\title{
A refined Hovmöller diagram
}

\author{
By OLIVIA MARTIUS*, CORNELIA SCHWIERZ and HUW C. DAVIES, \\ Institute for Atmospheric and Climate Science, ETH Zürich, 8092 Zürich, Switzerland
}

(Manuscript received 15 April 2005; in final form 24 October 2005)

\begin{abstract}
A refinement is proposed to the conventional Hovmöller diagram. The rationale is set out for the refined approach, a description provided of its design, and examples presented to illustrate its value as a diagnostic tool. It is shown that it can depict the dynamical characteristics of synoptic-scale disturbances as they evolve on a meandering tropopause-level waveguide, and in particular it can detect wave-breaking events, cut-off formation and vortex re-connection.
\end{abstract}

\section{Introduction}

A characteristic feature of upper-tropospheric flow is the presence of a meandering synoptic-scale wave pattern that tends to propagate eastwards within the ambient circumpolar flow. The pattern can constitute one component of the atmosphere's baroclinic wave structure and a localized large-amplitude lateral undulation is often accompanied by surface cyclogenesis. The pattern can also possess an embedded wave packet that can ultimately exert a strong influence upon the surface flow, and indeed such packets have been linked to the occurrence of extreme weather events (e.g. Langland et al., 2002; Grazzini and van der Grjin, 2003; Krishnamurti et al., 2003).

The deep baroclinic waves and accompanying cyclones traverse along the extratropical storm track established by the background flow's tropospheric thermal structure. The track has been termed the baroclinic waveguide by (Wallace et al., 1988) but equally can be viewed as a baroclinically determined waveduct formed by the tropopause-level enhanced isentropic PV-gradient and the surface baroclinicity. In contrast synoptic-scale disturbances, waves and wave-packets that are confined essentially to the tropopause propagate along the jet stream (Hoskins and Ambrizzi, 1993; Branstator, 2002), i.e. a spatially confined zone of enhanced isentropic potential vorticity (PV) gradient at that elevation (Massacand and Davies, 2001; Schwierz et al., 2004).

The quasi-horizontal zonal propagation of the aforementioned synoptic-scale waves of both classes mentioned above is commonly visualized using a Hovmöller diagram. It was originally developed to display the propagation of mid-tropospheric ridges and troughs (Hovmöller, 1949). In its conventional form the me-

\footnotetext{
*Corresponding author.

e-mail: olivia@env.ethz.ch

DOI: $10.1111 / \mathrm{j} .1600-0870.2006 .00172 . x$
}

teorological field adopted to examine the pattern (e.g. meridional velocity, geopotential height anomaly) is usually averaged over a pre-specified meridional band (e.g. $35^{\circ}-60^{\circ} \mathrm{N}$ ) and its zonal variation plotted against time (for an example see Chang, 1993, Fig. 2).

The technique is conceptually elegant and simple to apply, and it can provide a visual estimation of the zonal component of the phase and group velocity of individual wave peaks and wave trains. It has been used regularly since its introduction and recently it helped demonstrate zonal propagation of forecast errors (Langland et al., 2002).

There is, however, a significant drawback to the technique. The ambient deep-baroclinic or tropopause-confined waveguide is not necessarily zonally orientated (see e.g. Schwierz et al., 2004, Figs. 1 and 2). In effect wave propagation need not occur merely in the longitudinal direction, but for synoptic-scale waves is usually aligned along the meandering waveguide.

It would, therefore, be conceptually helpful to refine the Hovmöller diagram to capture the propagation along the spatially and temporally evolving waveguide itself rather than merely along a latitudinal belt. In this context Chang and Yu (1999) devised a Hovmöller diagram in a $20^{\circ}$ latitudinal band that varies longitudinally with a statistically determined climatological storm track but this procedure might preclude consideration of high amplitude or unusually shaped disturbances that, by their very nature, would be of significant interest.

In this paper a new method is proposed that refines the Hovmöller procedure to enable it to capture propagation along an instantaneous and space-time evolving PV waveguide at the tropopause. A detailed description of the method is provided in the next section, examples of its use as a diagnostic tool given in Section 3, and thereafter a critique of its merits and limitations is provided in the final section. 


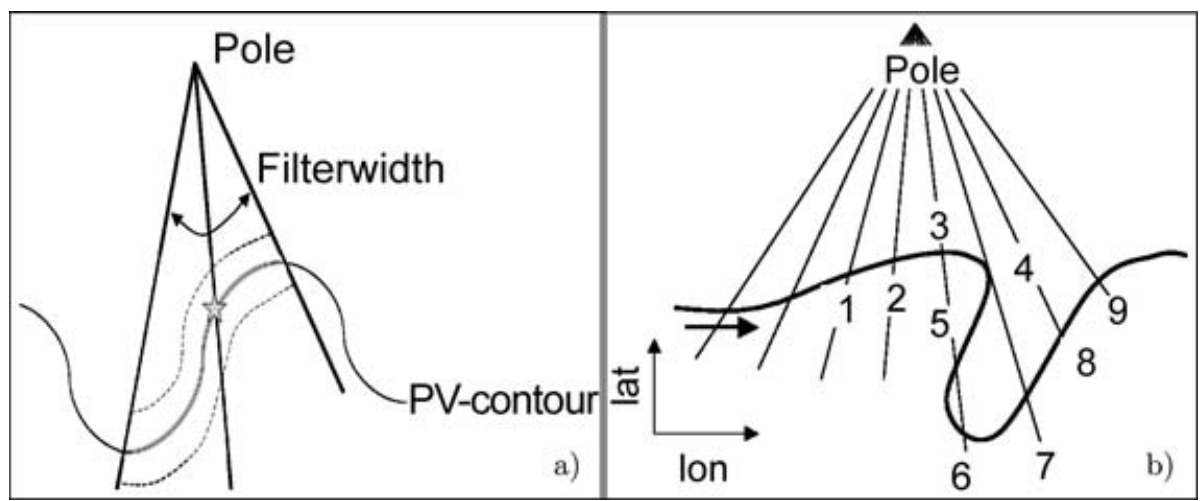

Fig. 1. Schematic depictions of the contour filtering method. (a) The evaluated longitude is marked with the star, the velocity is averaged within the band of $10^{\circ}$ width (dashed lines) around the contour in the filter area. (b) A situation with a wave-breaking contour. See text for details.

\section{Method}

The method is developed for, and illustrated using, a standard 4-D analysis (or forecast) set of an NWP suite and in theory allows to plot any desired 2-D field in the form of a Hovmöller diagram. In the present study use is made of the ERA-40 reanalysis fields of the European Centre for Mediumrange Weather Forecasts (ECMWF — see Simmons and Gibson, 2000). This data set covers the period from September 1957 to July 2002 and has a space-time resolution of respectively $1^{\circ}$ and 6 hours.

In a first step the location of the enhanced PV-gradient (i.e. waveguide) is determined for each time step. To this end we recognize that the jet stream at the tropopause break is co-located with the vertically and horizontally confined strong isentropic PV-gradient (e.g. Davies and Rossa, 1998). The hydrostatic isentropic form of the Ertel-PV is calculated on $\theta$-surfaces between 310 and $350 \mathrm{~K}$. The intersection of the dynamical tropopause, taken here as the $2 \mathrm{pvu}$ isosurface $\left[1 \mathrm{pvu}=10^{-6} \mathrm{Ks}^{-1} \mathrm{~kg}^{-1} \mathrm{~m}^{2}\right.$ ], with these isentropic surfaces is identified by determining on each isentropic surface the southernmost closed 2 pvu contour encompassing the pole. This contour is then adopted as the baseline for the evaluation of wave propagation.

The adoption of a single contour line ( 2 pvu line) is certainly convenient, but it is also based upon the expectation, supported by visual inspections and computation, that enhanced isentropic PV-gradients, if present, are located on the dynamical tropopause.

In a second step it is recognized that the length of the selected contour varies with time. For the purpose of constructing a Hovmöller-like diagram this variation is countered by introducing a normalized constant contour length. A filter is used to reduce the data along the contour to 360 values selected with a $1^{\circ}$ spacing. The filter is moved along the contour in steps of one degree longitude, and at each longitude the contour length is determined within a longitudinal sector of prespecified width (taken here as $8^{\circ}$, Fig. 1a). The variable tracked along the contour is then averaged within the filter over a latitu- dinal band of $10^{\circ}$ centered around the contour (dashed lines in Fig. 1a).

An example of how the area encompassed by the filter is determined is given next. First the intersections of the contour with longitudinal great circles of 1 degree spacing are determined (Fig. 1b). Then the intersection points of the filter bounds with the contour are determined for each longitude by moving anti-clockwise (west-east) along the contour with a stepsize of one degree. For example in Fig. 1b, assuming a filter width of 2 degrees, the filter at intersection nr.2 extends from intersection nr.1 to intersection nr.3. Sometimes multiple intersections of the contour with a longitudinal circle can occur, for example if the contour is S-shaped (e.g. intersections 3, 5, 6 in Fig. 1b). In this case the gridpoint furthest away eastwards from the base point along the contour is used to determine the filter extent. Hence the filter for intersection 3 reaches from intersection 2 to intersection 7. The filter for longitude 4 reaches from intersection 3 to intersection 8 . The next data point would be located at intersection 8 with a filter reaching from intersection 7 to intersection 9.

In a third step the variable selected for plotting is averaged within a band of $10^{\circ}$ centred around the contour inside the filter sector indicated as the area between the dashed lines in Fig. 1a. This procedure results in a single value per longitude and time step that is then plotted in the form of a longitude-time diagram.

Note that the choice of the filter width (opening angle of the filter) influences the smoothness of the wave features in the resulting Hovmöller diagram. In this study balance was sought between information loss due to smoothing and an overly noisy field due to very small-scale or spurious features. Tests were conducted with a wide range of filter widths and one appropriate value was found to be $8^{\circ}$.

Thus the procedure delivers two complementary data sets (i) the length of the contour in each filter band and (ii) the variation of the selected field along the contour reduced to 360 values. Intrinsic to the refined scheme's potential as a diagnostic tool is that space-time Hovmöller-like diagrams can be constructed from both these fields. 
Fig. 2. Hovmöller diagram of the length of the 2-pvu contour on the $320 \mathrm{~K}$ isentrope in November 1991, the contour length is normalised. The normalisation is done by dividing the contour length by the arc length encompassed by the filter on the latitudinal circle located at the mean latitude of the contour.

\section{Results and discussion}

In this section examples are provided to illustrate the diagnostic capabilities of the refined Hovmöller diagram.

\subsection{The contour-length Hovmöller diagram}

Consider now the Hovmöller-type diagram obtained by plotting the data for the contour length as a function of longitude. An example is given in Fig. 2 which shows the selected field's evolution for November 1991. To interpret the figure recall that a region of rapid temporal change in contour length denotes a high level of synoptic activity. In particular, a rapid increase can connote the development of a pronounced meridional orientation or even an S-shaped configuration of the contour, and hence betoken either the imminence or presence of wave breaking, i.e. irreversible deformation of the selected PVcontour (McIntyre and Palmer, 1984). Thus a rapid increase in contour length with the accompanying distortion of the waveguide is a dynamically significant event. Contrariwise in a subsequent phase the breaking wave can result in a cut-off in the form of an isolated disturbance (Appenzeller and Davies, 1992;

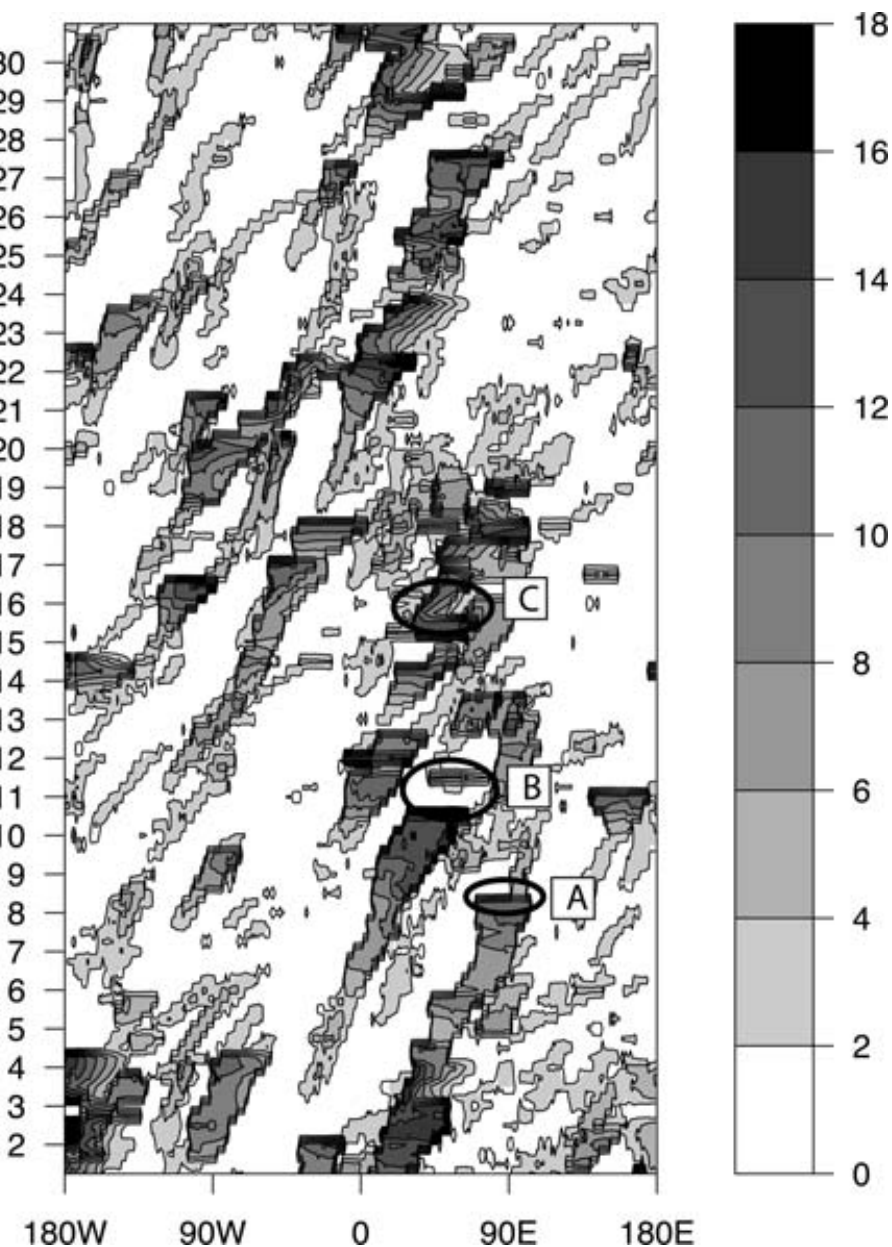

Thorncroft et al., 1993) with an attendant quantum decrease in the contour length. Alternatively the longitudinal extension of the breaking wave can drop beneath the horizontal resolution of the data.

In Fig. 2 events of breaking waves are discernible for example over the Himalayas on day 8 (marked by an A) and the formation of a cut-off that reconnects to the main stratospheric reservoir over eastern Europe on days 10, 11 and 12 (marked with a B).

It is evident that the contour-length-based diagram provides a concise depiction of deformation that on occasions leads to wave-breaking events. Also note that the diagram indicates that during November 1991 wave breaking occurred preferentially over Europe, where the prevailing climatological PV-gradients are weak. This is in good agreement with the findings of a climatological analysis of wave breaking using contour surgery (see Scott and Cammas, 2002, Fig. 10a).

\subsection{PV-Hovmöller diagrams}

Conventional and refined Hovmöller diagrams are juxtaposed in Fig. 3. The time period is again November 1991 and for sake of comparison the plotted field in both diagrams is the deviation 

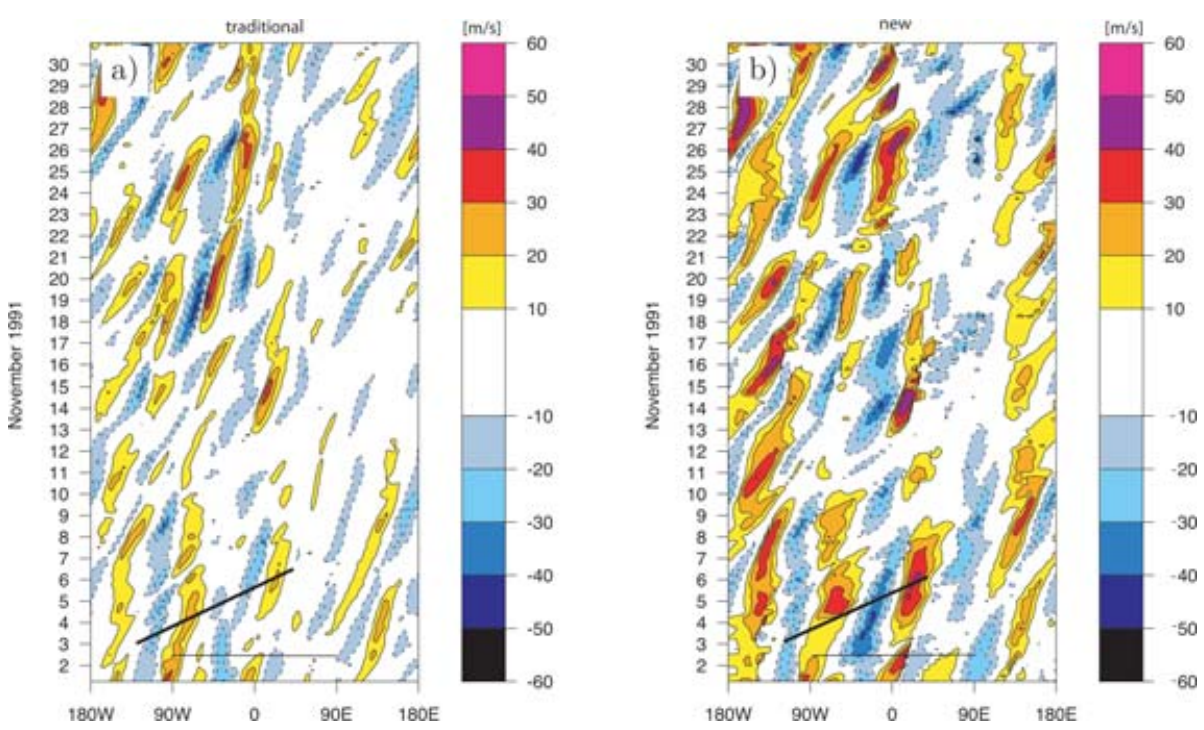

Fig. 3. Hovmöller diagrams of $v^{\prime}\left[\mathrm{m} \mathrm{s}^{-1}\right.$ ] in November 1991 on $320 \mathrm{~K}$ (a) calculated using the traditional method and (b) with the new method, contour interval is $10 \mathrm{~m} \mathrm{~s}^{-1}$. The thick black line indicates the wave train mentioned in the text, the thin horizontal line indicates the time and longitude section shown in Fig. 4.

of the meridional wind velocity from its climatological monthly mean value on the $320 \mathrm{~K}$ isentropic surface. For the conventional diagram the meridional wind velocity was averaged between 30 and $60^{\circ} \mathrm{N}$, and for the refined diagram a filter-width was adopted of $8^{\circ}$ and an averaged bandwidth of $10^{\circ}$.

The juxtaposition serves to illustrate the characteristics of the respective approaches. The two representations exhibit similar overall features including that of the approximate wave length, group velocity and phase velocity of the individual peaks. A notable difference is that the amplitudes of the wave peaks and troughs are often larger in the new representation. This difference is partly attributable to averaging over a larger band in the conventional diagram, but it can also be a direct consequence and intrinsic aspect of the new approach. In effect the refined approach entails limited meridional averaging about the centre of the waveguide where the amplitude of both the PV gradient and the accompanying velocity signal is expected to be largest. This limited meridional averaging contrasts with the $30^{\circ}$ used for the conventional method. It also follows that the amplitude displayed with the new representation reflects more accurately the strength of the in situ disturbances.

Closer inspection of the two plots in Fig. 3 reveals further differences. In the new representation a wave-train emanates from the mid Pacific (180W) around 3 November and reaches Europe on 7 November. In the conventional representation the corresponding and initially co-located train is interrupted at $50^{\circ} \mathrm{W}$. The absence of a negative peak at $50^{\circ} \mathrm{W}$ in the latter depiction can be explained by consulting Fig. 4 which shows that the associated peak is located between $60^{\circ}-70^{\circ} \mathrm{N}$ at $50^{\circ} \mathrm{W}$ on 2 November and will remain like that for the next two days (not shown).
This is too far polewards to be captured adequately with the $30^{\circ}-60^{\circ} \mathrm{N}$ meridional averaging used in the conventional Hovmöller diagram.

Another difference between the plots in Fig. 3 is evident on day 2 at $15^{\circ} \mathrm{E}$ where the time trace of the positive signal is vanishing in the conventional diagram. This discrepancy arises because the selected field possesses a rich meridional structure with the concurrent occurrence of negative and positive peaks at different meridional locations of the same longitude (see Fig. 4). In such a setting the conventional scheme's broad meridional averaging can and in this case does, extend over the opposing peaks and result in a destructive interference of the disturbance signal. It is worth noting that such a setting can be expected in a major wave-breaking event.

\section{Further remarks}

The examples provided in the previous section illustrated some of the merits of the refined Hovmöller diagram, and these derive essentially from representing disturbances as they evolve on a narrow undulating tropopause-level waveguide.

Here a brief critique is also provided of the new approach. First we note that a shortcoming of, or at least an interpretative challenge posed by, the new approach is associated with the combination of a complex flow evolution characterized by a highly distorted waveguide and the new scheme's spatial averaging procedure. An example is provided by the feature displayed in Fig. $3 \mathrm{~b}$ for 15 November at around $45^{\circ} \mathrm{E}$. It corresponds to a synoptic episode of a tropospheric cut-off that forms over eastern Europe/western Asia and rapidly reconnects back into the 


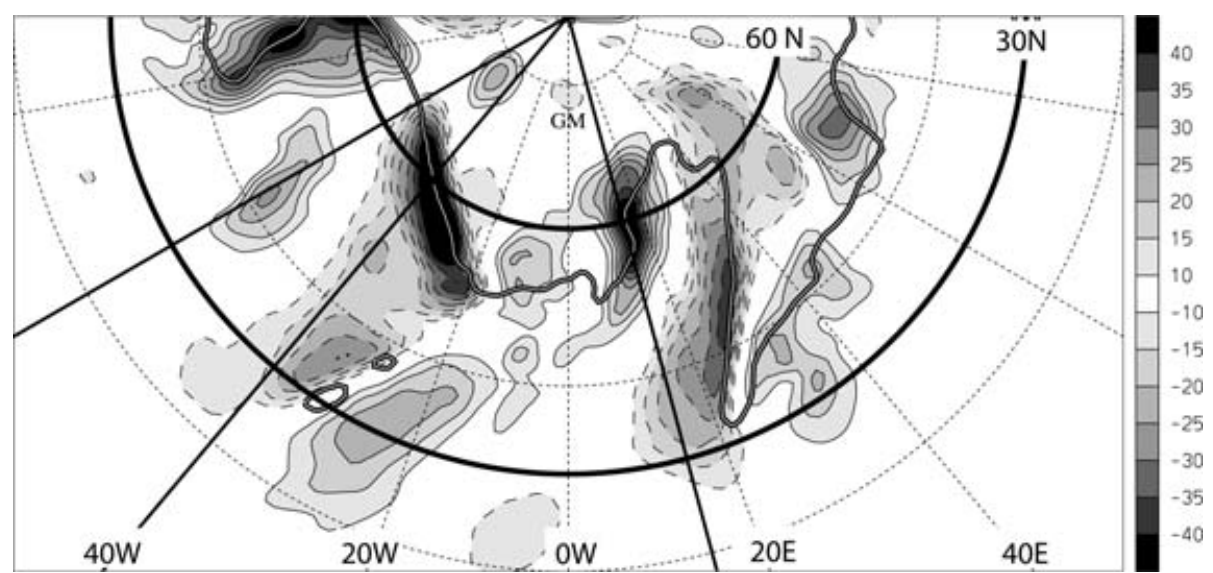

Fig. 4. $v^{\prime}\left[\mathrm{m} \mathrm{s}^{-1}\right]$ (shaded, negative dashed) and 2pvu contour (black-white line) on 320K on 2 November, 12 UTC. The coordinate grid has a resolution of $20^{\circ}$ and $30^{\circ} \mathrm{N}, 60^{\circ} \mathrm{N}, 60^{\circ} \mathrm{W}, 40^{\circ} \mathrm{W}$ and $15^{\circ} \mathrm{E}$ are indicated by thick black lines.

main tropospheric air mass. This sequence is accompanied by rapid variations in the contour length, and this variation is visible in the corresponding Hovmöller diagram of Fig. 2 (marked by C). Note also that an extant tropospheric (stratospheric) cut-off that had become far removed from the waveguide but then reemerged into the stratosphere (troposphere) would also produce a signal in the contour-length field. Thus a caveat is that synoptic experience is required to interpret some of the features captured in the refined Hovmöller plot.

A second caveat is also related to the complex structure of the tropopause-level flow. Comparison of the two types of Hovmöller diagrams reveals on occasions examples of comparatively space-time coherent peaks in the amplitude of waves or wave packets that are present in the conventional diagram but disappear in the refined diagram. (This is the obverse of the situation discussed in the final paragraph of the previous section). The inference is that the disturbance has remained within the zonal band sampled by the conventional approach but has been displaced away from the narrow band encasing the selected contour in the refined approach.

Further examination of such episodes confirms that the disturbances are no longer propagating along the selected 2-pvu contour on the specified isentropic surface, but induce wave propagation on another meridionally displaced tropopause-level band of enhanced isentropic PV-gradient located on a higher (lower) isentropic surface. (Note that if the latter waveguide was located outside the selected zonal-band of the conventional Hovmöller diagram it would also disappear from such a plot). The occurrence of multiple waveguides in the tropopause region can be expected from the existence of multiple jet streams (arctic, polar and subtropical jet, e.g. Shapiro and Keyser, 1990). A detailed study is currently underway to examine the dynamics of this 'waveguide to waveguide' transfer. In the present context the disappearance of the signal on the selected waveguide could be regarded as a limitation or shortcoming of the new approach. Alternatively it can be viewed as an indication of the new ap- proach's ability to detect the transfer of the disturbance signal away from the pre-selected waveguide.

Herein the emphasis has been on setting out the concept of and illuminating the use of the new diagram(s). However the method outlined in the previous section requires the à priori selection of a PV-contour and the specification of the parametersthe meridional band (set at $10^{\circ}$ ) and the longitudinal sector (set at $8^{\circ}$ ). For the widths of the meridional and longitudinal bands sensitivity studies indicate the appropriateness of the $10^{\circ}$ and $8^{\circ}$ values (Martius, 2005). Clearly the existence of multiple waveguides alluded to above implies that a Hovmöller diagram could be constructed for each waveguide and the interwaveguide transfer illustrated by co-displaying the resulting diagrams.

In summary we conclude that the refined Hovmöller diagrams capture the essence of disturbance propagation, amplification, and disappearance on a tropopause-level waveguide. The conventional and refined diagrams show good overall agreement of such wave features as wavelength, group and phase velocities, but significant differences arise in complex flow evolutions such as wave breaking and the re-emergence of cut-off features. Also the new approach delivers additional information on the length of seminal PV contours at each longitude interval and this enables the detection of wave-growth, wave breaking and re-emergence events. In principle it should be possible to generate a wave-breaking index based on the temporal variation of the contour length and thereby assemble a climatological data set of the spatial and temporal distribution of breaking waves along the extratropical tropopause. The refined diagram also prompts consideration of the presence, influence and dynamics of multiple waveguides.

\section{Acknowledgments}

The authors would like to thank MeteoSchweiz for providing access to the ERA40 data set. This research is funded by the 
NCCR-Climate program. Cordial thanks to Heini Wernli for providing the contour identification routine.

\section{References}

Appenzeller, C. and Davies, H. C. 1992. Structure of stratospheric intrusions into the troposphere. Nature 358, 570-572.

Branstator, G. 2002. Circumglobal teleconnections, the jet stream waveguide, and the North Atlantic oscillation. J. Clim. 15, 1893-1910.

Chang, E. K. M. 1993. Downstream Development of Baroclinic Waves as Inferred from Regression-Analysis. J. Atmos. Sci. 50, 2038-2053.

Chang, E. K. M. and Yu, D. B. 1999. Characteristics of wave packets in the upper troposphere. Part I: Northern Hemisphere winter. J. Atmos. Sci. 56, 1708-1728.

Davies, H. C. and Rossa, A. M. 1998. PV Frontogenesis and UpperTropospheric Fronts. Mon. Wea. Rev. 126, 1528-1539.

Grazzini, F. and van der Grjin, G. 2002. Central European Floods during summer 2002. ECMWF Newsletter 96, 18-28.

Hoskins, B. J. and Ambrizzi, T. 1993. Rossby-wave propagation on a realistic longitudinally varying flow. J. Atmos. Sci. 50, 1661-1671.

Hovmöller, E. 1949. The trough and ridge diagram. Tellus 1, 62-66.

Krishnamurti, Y., Vijaya Kumar, T. S. V., Rajendran, K. and Hopkins, A. 2003. Antecedents of the flooding over south-eastern England during October 2000. Weather 58, 367-370.
Langland, R. H., Shapiro, M. A. and Gelaro, R. 2002. Initial condition sensitivity and error growth in forecasts of the 25 January 2000 East Coast snowstorm. Mon. Wea. Rev. 103, 957-974.

Martius, O. 2005. Climatological Aspects of Wave Disturbances on the Tropopause and Links to Extreme Weather in Europe. PhD-Thesis No 16152, ETH Zurich, Switzerland.

Massacand, A. C. and Davies, H. C. 2001. Interannual variability of the extratropical northern hemisphere and the potential vorticity wave guide. Atmos. Sci. Lett. doi:10.1006/asle.2001.0027.

McIntyre, M. E. and Palmer, T. N. 1984. The 'surf-zone' in the stratosphere. Atm. and Terr. Phys. 46, 825-849.

Schwierz, C., Dirren, S. and Davies, H. C. 2004. Forced waves on a zonally aligned jet stream. J. Atmos. Sci. 61, 73-87.

Shapiro, M. A., and, Keyser, D. 1990. Fronts, Jet Streams and the Tropopause. In: Extratropical Cyclones, Palmén Memorial Volume (eds. C. Newton and E. Holopainen), Amer. Meteor. Soc., 167-191.

Simmons, A. J., and Gibson, J. K. 2000. The ERA-40 Project Plan. Technical Report, ECMWF Shinfield Park Reading UK.

Scott, R. K., and Cammas, J. P. 2002. Wave breaking and mixing at the subtropical tropopause. J. Atmos. Sci. 59, 2347-2361.

Thorncroft, C. D., Hoskins, B. J. and McIntyre, M. E. 1993. Two Paradigms of Baroclinic-Wave Life-Cycle Behavior. Quart. J. Roy. Meteoro. Soc. 119, 17-55.

Wallace, J. M., Lim, G. H. and Blackmon, M. L. 1988. Relationship between cyclone tracks, anticyclone tracks and baroclinic wave-guides. J. Atmos. Sci. 45, 439-462. 